\title{
How accurate is the 'Surprise Question' at identifying patients at the end of life? A systematic review and meta-analysis
}

\author{
Nicola White ${ }^{*}$ D, Nuriye Kupeli, Victoria Vickerstaff and Patrick Stone
}

\begin{abstract}
Background: Clinicians are inaccurate at predicting survival. The 'Surprise Question' (SQ) is a screening tool that aims to identify people nearing the end of life. Potentially, its routine use could help identify patients who might benefit from palliative care services. The objective was to assess the accuracy of the SQ by time scale, clinician, and speciality.

Methods: Searches were completed on Medline, Embase, CINAHL, AMED, Science Citation Index, Cochrane Database of Systematic Reviews, Cochrane Central Register of Controlled Trials, Open Grey literature (all from inception to November 2016). Studies were included if they reported the SQ and were written in English. Quality was assessed using the Newcastle-Ottawa Scale.

Results: A total of 26 papers were included in the review, of which 22 reported a complete data set. There were 25,718 predictions of survival made in response to the SQ. The c-statistic of the SQ ranged from 0.512 to 0.822 . In the meta-analysis, the pooled accuracy level was $74.8 \%(95 \% \mathrm{Cl} 68.6-80.5)$. There was a negligible difference in timescale of the SQ. Doctors appeared to be more accurate than nurses at recognising people in the last year of life (c-statistic $=0.735$ vs. 0.688), and the SQ seemed more accurate in an oncology setting $76.1 \%$ (95\% Cl 69.7-86.3).

Conclusions: There was a wide degree of accuracy, from poor to reasonable, reported across studies using the SQ. Further work investigating how the SQ could be used alongside other prognostic tools to increase the identification of people who would benefit from palliative care is warranted.
\end{abstract}

Trial registration: PROSPERO CRD42016046564.

Keywords: Surprise question, Accuracy, Prognosis, End of life, Palliative care, Survival

\section{Background}

In both the UK and the USA, the Surprise Question (SQ; "Would you be surprised if this patient died within the next $\times$ months?") has been suggested as a trigger for referral to specialist palliative care $[1,2]$.

It has been estimated that between $69 \%$ and $82 \%$ of dying patients in the UK would benefit from palliative care input (specialist or generalist) [3]. There were approximately $160,000-170,000$ referrals made to specialist palliative care services in the UK between 2008 and 2009 [4]. In the USA, it has been estimated that between

\footnotetext{
* Correspondence: n.g.white@ucl.ac.uk

Marie Curie Palliative Care Research Department, Division of Psychiatry, University College London, 6th Floor, Maple House, 149 Tottenham Court Road, London W1T 7NF, UK
}

1 million and 1.8 million (7.5\% and 8.0\%) of hospital admissions have a palliative care need [5].

It has been repeatedly shown that clinicians are inaccurate at prognostication [6-8] and in recognising dying patients [9]. Therefore, there is an increased likelihood that patients who would benefit from palliative care are potentially being missed because validated prognostic tools (e.g. Palliative Prognostic Score [10] or Palliative Prognostic Indicator [11]) are not being used routinely, either due to their perceived complexity or inconvenience [12].

The SQ offers an alternative to a standard prognostic estimate. It does not require clinicians to collect clinical data or to use a scoring algorithm, nor does it require clinicians to make a specific estimate about length of 
survival; it simply asks whether the respondent would be surprised if the patient were to die within a specified time period (usually the next year). It was originally developed by Joanne Lynn as a method to identify patients who might benefit from palliative care services [13], asking the clinician: "Is this person sick enough that it would be no surprise for the person to die within the next 6 months, or a year?" Since its development, the $\mathrm{SQ}$, or variants thereof, have been incorporated into clinical guidelines such as National Institute for Health and Care Excellence (NICE) for End of Life Care [14], and adopted into routine clinical practice in various settings, including hospitals, hospices, and General Practices. Although developed as a standalone item, it is now included as part of the Gold Standard Framework (GSF) proactive identification guidance tool in the UK [1], in which clinicians are encouraged to ask themselves "Would you be surprised if this patient were to die in the next 6-12 months". A response of "No" to the SQ may trigger a referral to specialist palliative care services, or to the adoption of a palliative care approach to future care. This parsimonious approach could potentially identify more patients who need palliative care and could be incorporated into routine clinical practice with relative ease and at little or no extra cost.

Yet, how accurate or effective is the SQ at identifying people in the last year of life? Could it be used to identify people who are just days from dying? Is one clinical group more accurate at using the SQ than another? Is the SQ more accurate when used by one professional group rather than another? The present study aims to assess the accuracy of the SQ by time scale, clinician, and speciality.

\section{Methods}

\section{Data sources and searches}

We initially searched the literature using the terms, "surprise question" and "death". Medline, Embase, CINAHL, AMED, Science Citation Index, Cochrane Database of Systematic Reviews, and Cochrane Central Register of Controlled Trials databases were searched. All databases were searched from inception up to the date of the search (November 2016), including papers still being processed by the databases (for exact search terms, see Table 1). In addition, the references of all included studies were checked and authors of papers were contacted to check for any additional papers and for full papers if only an abstract was identified. After contacting the authors of identified papers, it was discovered that our original search strategy had failed to identify one key paper [15]; therefore, the search terms were amended to include "GSF" as a keyword. The search strategy was then re-run on all databases.
Table 1 Search Strategy

\begin{tabular}{|c|c|c|c|}
\hline Database & Search Terms & $\begin{array}{l}\text { \# papers } \\
\text { search } 1 \\
\text { (Aug 2016) }\end{array}$ & $\begin{array}{l}\text { \# papers } \\
\text { search } 2 \\
\text { (Nov 2016) }\end{array}$ \\
\hline $\begin{array}{l}\text { OVID platform: Medline, } \\
\text { Embase, AMED }\end{array}$ & $\begin{array}{l}\text { Dying.mp. } \\
\text { Death.mp. } \\
\text { mortality.mp. } \\
1 \text { or } 2 \text { or } 3 \\
\text { (surprise adj1 } \\
\text { question).mp. } \\
\text { Gsf.mp } \\
5 \text { or } 6 \\
4 \text { and } 7\end{array}$ & 55 & 137 \\
\hline Web of Science & $\begin{array}{l}\text { TS = (surprise NEAR/1 } \\
\text { question OR GSF) } \\
\text { TS = (dying OR death } \\
\text { OR mortality) } \\
\# 1 \text { AND \#2 }\end{array}$ & 31 & 68 \\
\hline CINAHL (EBSCOhost) & $\begin{array}{l}\text { TX surprise question } \\
\text { TX GSF } \\
\text { TX dying } \\
\text { TX mortality } \\
\text { TX death } \\
3 \text { OR } 4 \text { OR } 5 \\
1 \text { OR } 2 \\
6 \text { AND } 7\end{array}$ & 13 & 33 \\
\hline $\begin{array}{l}\text { Database of systematic } \\
\text { reviews and Cochrane } \\
\text { Central Register of } \\
\text { Controlled Trials }\end{array}$ & $\begin{array}{l}\text { surprise NEAR/1 } \\
\text { question } \\
\text { TX GSF } \\
\text { TX dying } \\
\text { TX mortality } \\
\text { TX death } \\
3 \text { OR } 4 \text { OR } 5 \\
1 \text { OR } 2 \\
6 \text { AND } 7\end{array}$ & 8 & 12 \\
\hline Open Grey & "Surprise Question" & 0 & 0 \\
\hline
\end{tabular}

GSF Gold Standard Framework, TS topic, TX all text

\section{Study selection}

All study designs were included.

\section{Inclusion}

- Studies conducted in human subjects

- Studies reporting the use of the SQ

\section{Exclusion}

- Studies which did not quantify the accuracy of the SQ (or for which this information was not available from study authors)

- Studies that collected data retrospectively

- Not reported in English

Originally, it was planned to exclude all studies that were in abstract form. However, due to the low number of studies initially identified, and the relative low risk associated with including such data, we opted to be inclusive of all studies. We contacted all authors of abstracts to obtain a full study report. If a full study was not 
available, abstracts from which relevant data could be extracted were included.

\section{Quality assessment}

The quality of studies was assessed with the NewcastleOttawa Scale [16]. This scale was selected due to the nature of the studies included (non-randomised controlled trial, observational). The raters met and discussed each domain of the scale, completed one study using the scale, and discussed any discrepancies or difficulties that were identified before completing the assessment on all studies. In order to provide a full account of the accuracy of the SQ, no study was excluded based on the risk of bias score alone; however, if possible, it was planned to undertake a sensitivity analysis to account for the effect of poor quality studies. Each paper was assessed by two individual raters (NW and NK). Any discrepancies were resolved by a meeting of the two reviewers, with a third reviewer (PS) being included if the discrepancy was unresolved.

\section{Selection}

The studies identified from the database searches were screened by two reviewers independently (NW and NK). The first selection criterion was that the abstract/title included the use of the SQ. Any study not meeting this criterion was excluded. At full review, studies were selected for inclusion if they reported a quantifiable measure of the accuracy of the SQ (e.g. the proportion of patients correctly identified as being in the last year of life, as opposed to a qualitative assessment such as "the SQ performed well"). Any discrepancies at either selection point were resolved by a meeting between the two reviewers. If unresolved, a third reviewer (PS) was consulted.

\section{Extraction}

The following data were extracted from each paper:

- A description of the study population (both patient and clinician)

- The format of the SQ that was used in the study (e.g. the length of time to which the SQ related, or whether the SQ referred to expected survival or expected death)

- The accuracy of the SQ (i.e. how many people who were identified as likely to die, did actually die)

\section{Data synthesis and analysis}

A narrative synthesis of the findings from the studies was completed. This included details about the patient population characteristics, clinician characteristics, the format of the SQ, and the outcome (accuracy of SQ).
A quantitative synthesis was completed from those studies where data were available. All authors were contacted if the published data were incomplete or where calculation errors were noted in the published manuscript. Stata v13.0 was used for all analyses.

The accuracy of the SQ was summarised in a $2 \times 2$ table for each study. The sensitivity (the ability to recognise those who were dying), specificity (the ability to recognise those who were not dying), positive predictive value (PPV, the proportion of patients who died when the clinician predicted dying), and the negative predictive value (NPV, the proportion of patients who survived when the clinician predicted survival) were calculated [17]. The area under the curve (AUROC), also known as the c-statistic value, was calculated. This statistic compares the number of correct estimates (sensitivity) against the number of false estimates (1-specificity). A score of 0.5 indicates a model with poor predictive value, meaning that clinicians are no better than chance at identifying a person nearing the end of their life. An increase in the value (to a maximum score of 1 ) indicates an increase in the level of clinician accuracy.

The accuracy overall, by timeframe, by profession, and by speciality, was calculated by meta-analysis, using the "metaprop" command in Stata. These data were used to assess heterogeneity of the data synthesis using the $I^{2}$ statistic. Where possible, a sub-analysis of accuracy by clinician profession, patient group, and clinical setting were completed. To account for the $0 \%$ and $100 \%$ limits, the data obtained were transformed using the Freeman Tukey double arcsine method, and a meta-analysis was completed using the DerSimonian-Laird method with inverse variance weighting and then back transformed to present the percentage accuracy. We examined the impact of the time-frame of the SQ on its diagnostic accuracy. For this analysis, the time frame was categorised into up to 30 days, up to 6 months, and up to 12 months.

Publication bias was assessed using a funnel plot (Additional file 1: Figure S1).

\section{Role of the funding source}

This review was completed as part of a $\mathrm{PhD}$ studentship awarded from University College London. The funders had no role in this systematic review.

\section{Results}

In total, 357 studies were identified from the database searches (Fig. 1). No studies were identified through the grey literature search. Of those studies initially identified, 182 were subsequently found to be duplicates. There were 175 studies that were screened by title and abstract, of which 118 were excluded. Of the 57 full text articles retrieved, 34 were excluded for various reasons 


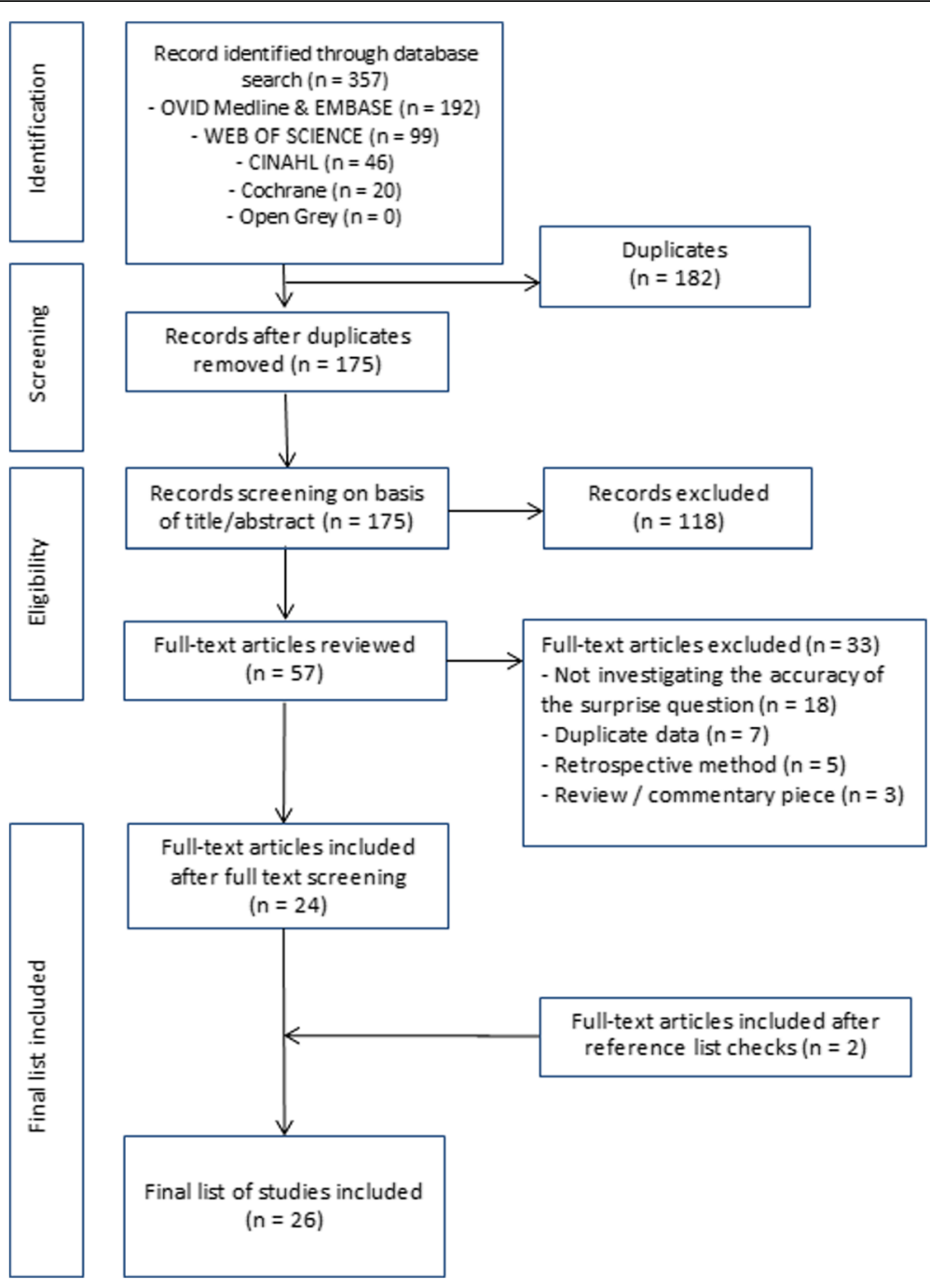

Fig. 1 PRISMA flowchart

(Additional file 2: Table S1). Three additional studies were identified from a search of the references of the included studies. In total, 26 papers were included in this review [15, 18-42].

We were able to obtain data from one unpublished paper with which we replaced one of the abstracts [33]. Another abstract [41] was excluded because the full paper had also been published [43].

Each paper included underwent a quality assessment with high agreement between the two raters (ICC 0.93) (Additional file 3: Table S2). No paper was excluded on the basis of their quality score.

A summary of the studies included can be seen in Table 2. Of the 26 papers, ten (38\%) were from the UK and nine $(35 \%)$ were from the USA. Ten $(38 \%)$ of the studies were presented in abstract form, the rest were full papers. Eight of the included studies related to patients with end-stage renal disease, six related to cancer patients, four related to patients with heart failure, one study related to patients with sepsis, one study related to chronic obstructive pulmonary disease and six studies included patients with a variety of different diagnoses. The majority of studies $(15,58 \%)$ specified 12 months as the relevant period for the SQ. Eight papers specified a time frame of 6 to 12 months. Three papers specified shorter time periods.

We were able to extract data from $22 / 26$ papers either directly from the paper or after contacting the author (Table 3).

\section{Accuracy of the SQ}

The outcomes of 25,718 estimates were reported in the 22 studies with complete data. Patients died within the specified timeframe (whatever it was in that particular study) on 4217 occasions (16\%). A response of "No, I would not be surprised" was given on 6495 occasions 


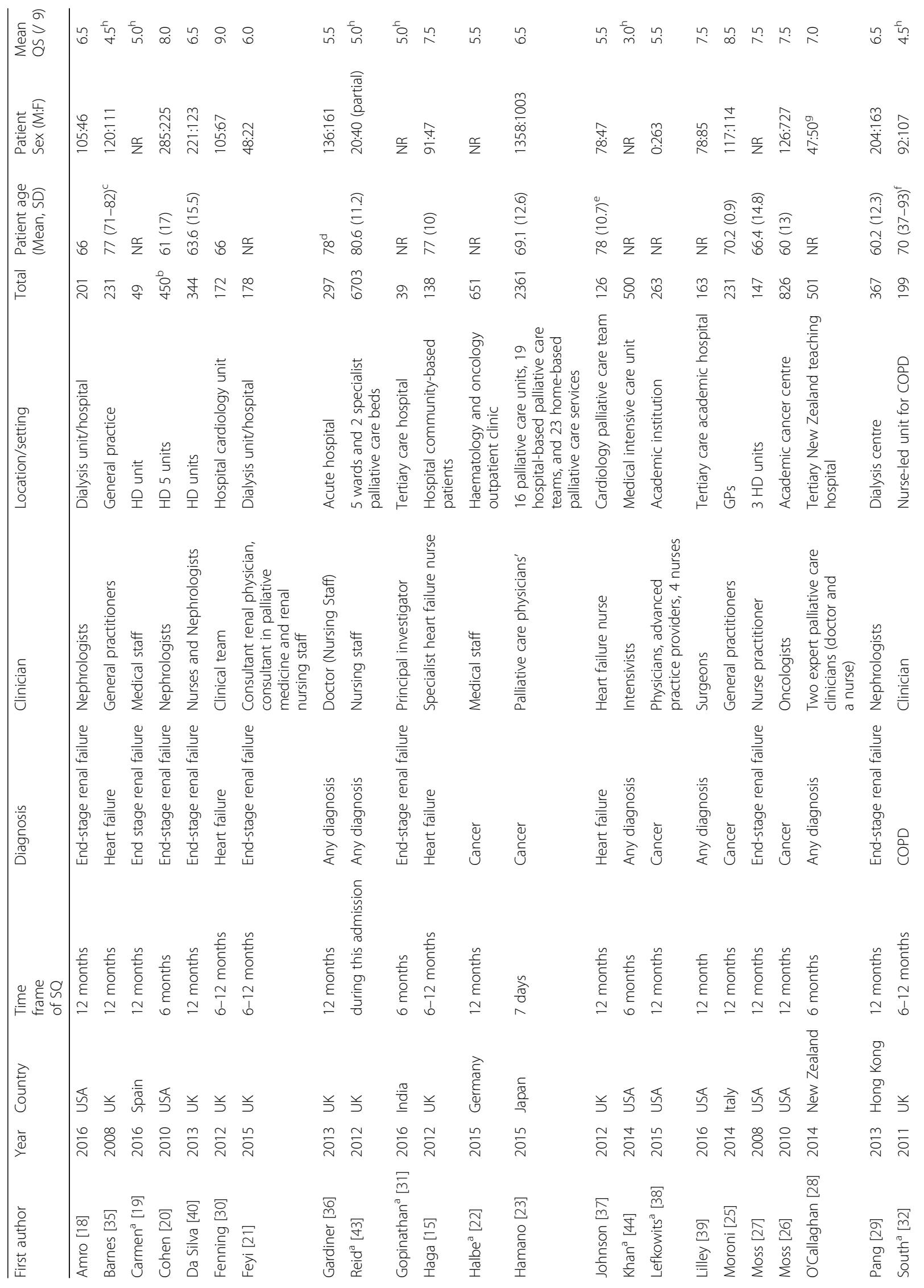




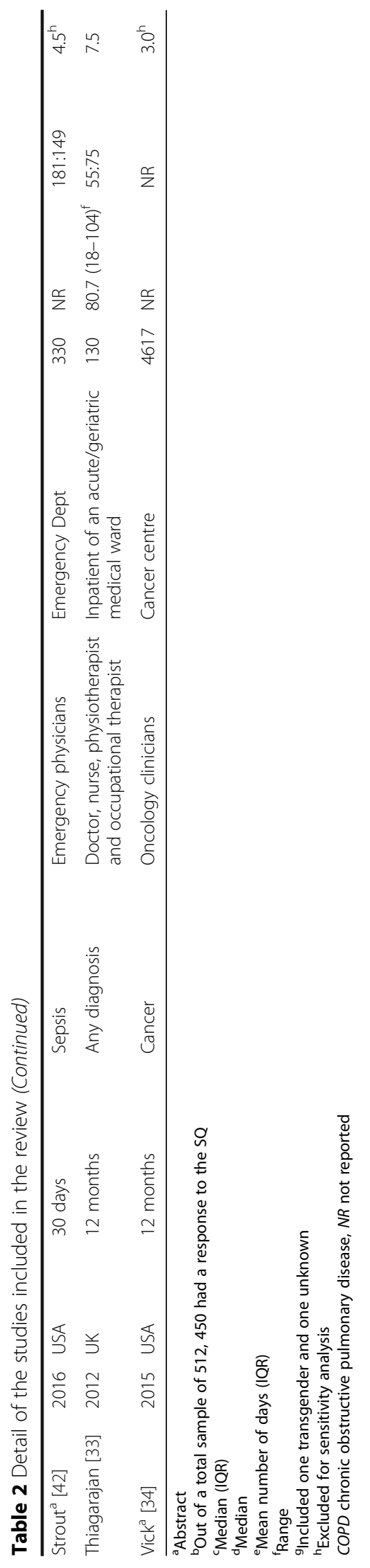




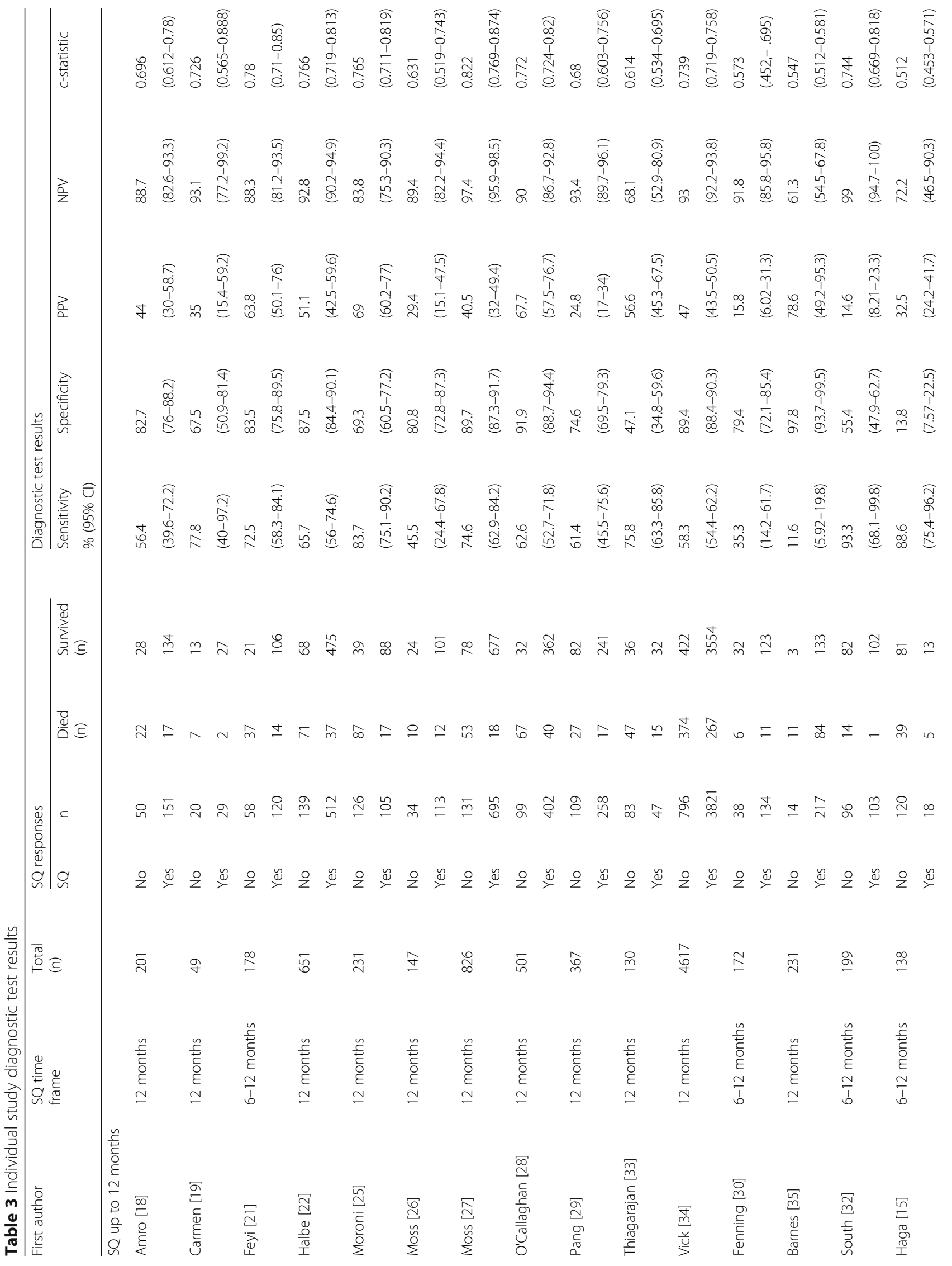




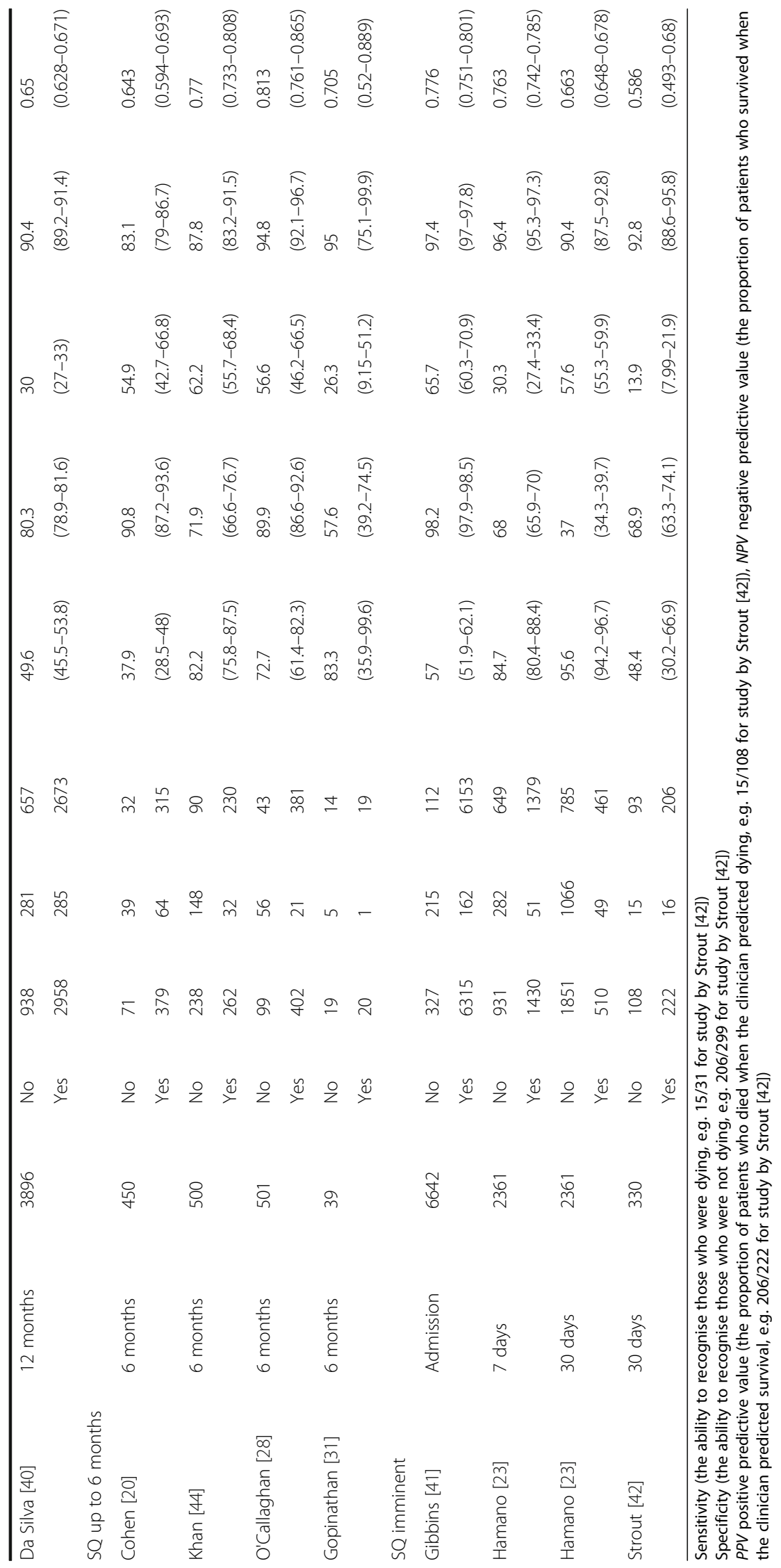


(25\%) and clinicians' intuitions were 'correct' (about whether they should or should not be surprised) in 20,964/25,718 cases (82\%). Most of the correct attributions occurred when clinicians indicated that they would be surprised if the patient died within the specified time period (19,223 occasions) and they did in fact survive for that length of time (17,985 occasions).

The results across the studies (Table 3) showed a wide variation in the reported accuracy of the SQ. The sensitivity ranged between $11.6 \%$ and $95.6 \%$ and a range of $13.8 \%$ to $98.2 \%$ was reported for specificity. The PPV ranged from $13.9 \%$ to $78.6 \%$, and the NPV ranged from $61.3 \%$ to $99 \%$. The AUROC score (c-statistic) across the studies ranged from 0.512 to 0.822 .

There was no indication of publication bias from the funnel plot (Additional file 1: Figure S1).

On meta-analysis, the pooled level of accuracy, that is the number of times the clinician correctly predicted the outcome of a patient, was 74.8\% (95\% CI 68.6-80.5; $I^{2}=$ $99.1 \%, 95 \%$ CI 99-99). The studies were sorted by date of publication and there appeared to be no trend by year (Fig. 2). After a sensitivity analysis for lower quality rated scores, in which eight papers were removed $[19,31,32$, $34,35,41,42,44]$, the pooled accuracy level increased to 75.4\% (95\% CI 70.8-79.7; $I^{2}=96.8,95 \%$ CI 96-98). Those studies that used a shorter time frame for the SQ (up to 6 months) had a pooled estimate of $76.6 \%$ (95\% CI 61.6-88.8\%; $I^{2}$ 99.6\%, 95\% CI 100-100), and when the time frame was reduced to imminent death (i.e. 7 days, 30 days or 'this admission') the pooled accuracy estimate was 76.4\% (95\% CI 52.4-93.8; $I^{2}$ 99.8\%, 95\% CI

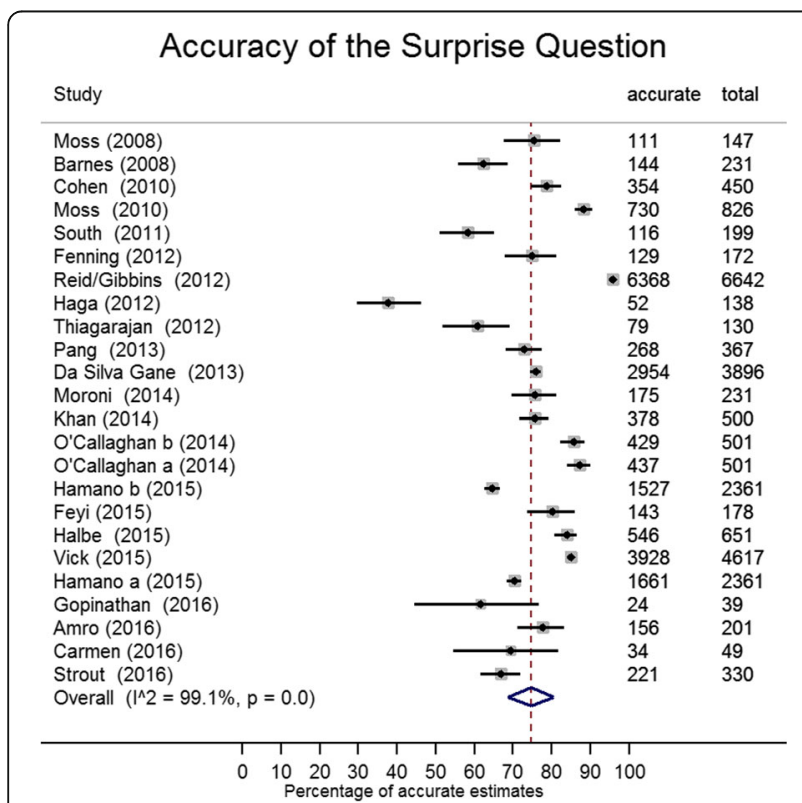

Fig. 2 Proportionate data of the number of correct estimates out of the total number of responses
100-100) (Fig. 3). The meta-regression indicated that the increase in time frame did not impact on the diagnostic accuracy of the SQ: comparing up to 30 days with 12 months (difference in accuracy $=0.8 \%, 95 \% \mathrm{CI}-12.8$ to $14.5, P=$ 0.901 ) and comparing up to 6 months with 12 months (difference $=4.3 \%, 95 \% \mathrm{CI}-10.8$ to $19.4, P=0.561$ ).

One unpublished paper [33] reported the results of the SQ and a modified version of the $\mathrm{SQ}$, by asking clinicians "would you be surprised if this person was to be alive in a year's time?" By rewording the question, they found that specificity was improved (i.e. correctly identifying those who do not die) but sensitivity was reduced (i.e. less correct predictions about those who will die).

\section{The SQ by profession and by specialty}

One paper reported the difference in performance of the SQ when used by nurses and doctors [40]. As a result of additional data supplied by the author, it was possible to calculate the sensitivity, specificity, PPV, and NPV for both doctors and nurses (Table 4).

Doctors appeared to be better at predicting dying within 12 months, with a sensitivity of $73 \%$ (95\% CI $63-$ 82 ) and specificity of $74 \%$ (95\% CI 70-78) compared to a sensitivity of $45 \%$ (95\% CI $40-49)$ and specificity of $82 \%$ (96\% CI $80-83$ ) for nurses. The c-statistic for doctors was 0.735 (95\% CI $0.688-0.783)$ compared to 0.632 (95\% CI 0.608-0.655) for nurses.

Of the 22 papers that included data, eight reported research conducted within a haemodialysis context [18-21, 27, 29, 31, 40], five were within oncology $[22,23,25,26,34]$, and nine papers were on other areas (five on all diagnoses [28, 33, 35, 41, 44], one on sepsis [42], two on heart disease [15, 30], and one on COPD [32]). Table 5 highlights the range of scores from the individual studies reporting from each specialty.

On meta-analysis, the pooled accuracy for oncology was 78.6\% (95\% CI 69.7-86.3; $I^{2}=99.0 \%$, 95\% CI 99-99). The pooled accuracy for renal was $76.1 \%$ (95\% CI 73.9$78.3 \% ; I^{2}=36.0 \%, 95 \%$ CI $0-72$ ). The pooled accuracy estimate for the other group was $72.3 \%$ (95\% CI 58.0 84.6; $I^{2}=99.1 \%$, 95\% CI 99-99) (Fig. 4).

\section{Studies with incomplete data}

Four studies had insufficient data to be included in the meta-analysis. Three authors responded to requests, but were unable to provide additional data to that presented in the paper [37, 39]. One author was not contactable [38].

One study [37] did not report data on the accuracy of a 'Yes' response to the SQ. In this study, it was reported that specialist heart failure nurses would not be surprised if 88/126 patients would have died within 12 months. In fact, 78/88 (89\%) of those patients did die 


\section{Accuracy of the surprise question}

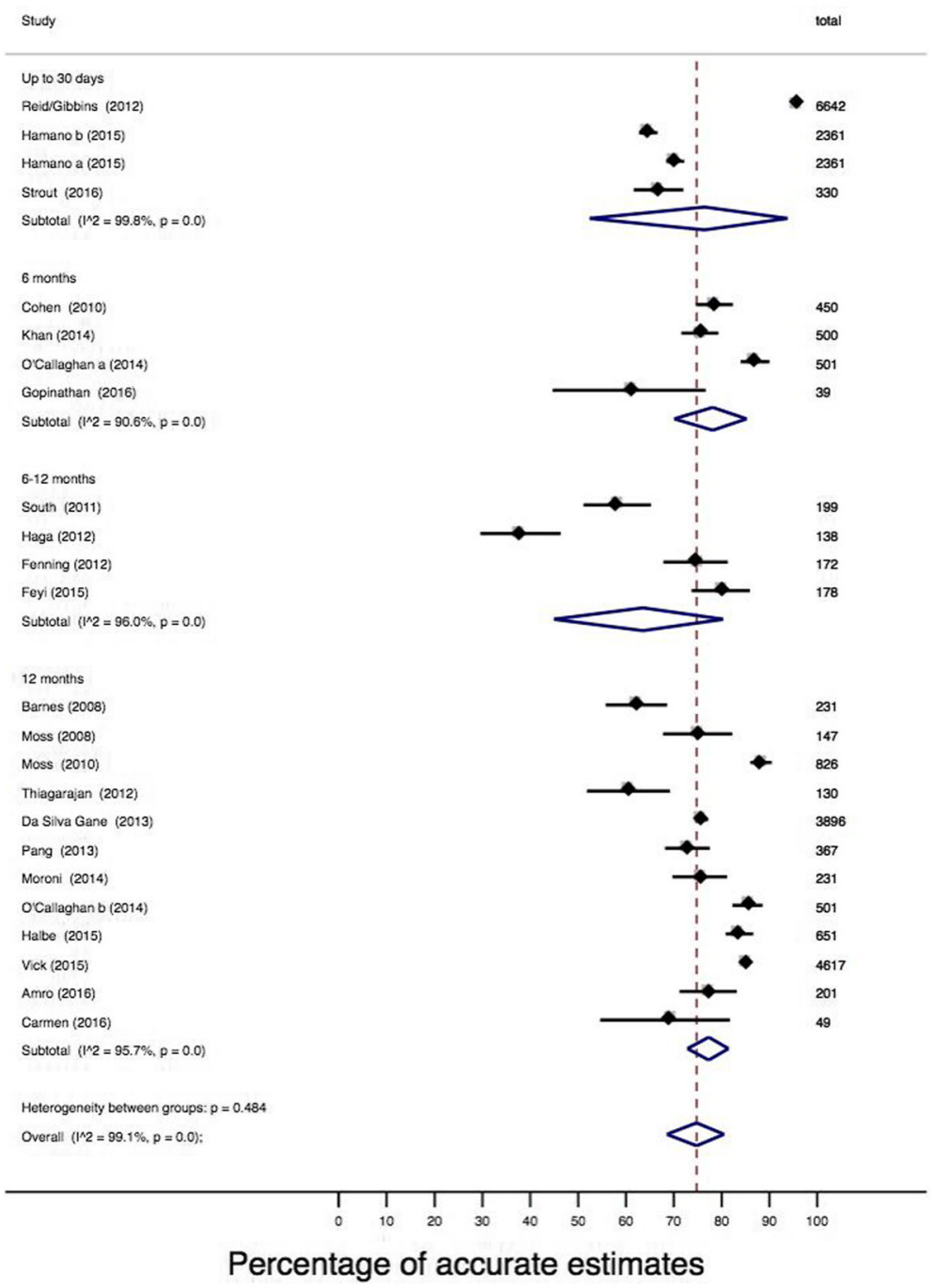

Fig. 3 Meta-analysis of the accuracy of the surprise question by time frame

Table 4 Surprise Question accuracy by profession

\begin{tabular}{|c|c|c|c|c|c|c|c|c|}
\hline Total & & Died & Survived & Sensitivity & Specificity & PPV & NPV & c-statistic \\
\hline \multicolumn{9}{|c|}{ Doctors } \\
\hline No & 218 & 71 (33) & 147 (67) & & & & & \\
\hline Yes & 442 & $26(6)$ & 416 (94) & 73 & 74 & 33 & 94 & 0.735 \\
\hline Total & 660 & $97(15)$ & $563(85)$ & $(63-82)$ & (70-78) & (26-39) & $(92-96)$ & $(0.688-0.783)$ \\
\hline \multicolumn{9}{|c|}{ Nurses } \\
\hline No & 720 & 210 (29) & $510(71)$ & & & & & \\
\hline Yes & 2516 & 259 (10) & 2257 (90) & 45 & 82 & 29 & 90 & 0.632 \\
\hline Total & 3236 & 469 (14) & 2767 (86) & $(40-49)$ & (80-83) & (26-33) & (86-91) & $(0.608-0.655)$ \\
\hline
\end{tabular}

Sensitivity (the ability to recognise those who were dying, e.g. 71/97 for doctors)

Specificity (the ability to recognise those who were not dying, e.g. 26/97 for doctors)

$P P V$ positive predictive value, the proportion of patients who died when the clinician predicted dying, e.g. 71/218 for doctors), NPV negative predictive value, the proportion of patients who survived when the clinician predicted survival, e.g. 416/442 for doctors) 
Table 5 Diagnostic scores of the accuracy of the Surprise Question across specialties

\begin{tabular}{|c|c|c|c|c|c|c|c|}
\hline & Studies & Estimates & PPV & NPV & Sensitivity & Specificity & c-statistic \\
\hline & (n) & (n) & Mean (SD), Range & Mean (SD), Range & Mean (SD), Range & Mean (SD), Range & Mean (SD), Range \\
\hline Oncology & 6 & 11,047 & 49.3 (13.5), 30.3-69 & $92.3(4.9), 83.8-97.4$ & 77.1 (13.7), 58.3-95.6 & 73.5 (20.5), 37-89.7 & $0.753(0.052), 0.663-0.822$ \\
\hline Renal & 8 & 5327 & 38.5 (14.4), 24.8-63.8 & $90.2(3.8), 83.1-95$ & 60.6 (16.2), 37.9-83.3 & $77.2(10.4), 57.6-90.8$ & 0.689 (0.049), $0.631-0.78$ \\
\hline Other & 10 & 9344 & 46.4 (24.5), 13.9-78.6 & 85.5 (13.3), 61.3- 99 & $62.8(25.5), 11.6-93.3$ & 71.4 (26.7), 13.8-98.2 & $0.671(0.114), 0.512-0.822$ \\
\hline
\end{tabular}

PPV positive predictive value, NPV negative predictive value

within 12 months. However, no data were reported about the outcomes for the 38 patients where the heart failure nurses would have been surprised if the patient had died. One study only contained data relating to the responses from the clinicians but not the outcome [36]. Gardiner et al. (2013) described the responses from doctors and nurses for two survival time points: 12 months and "death during this admission". Of 297 patients, doctors would not have been surprised if 123 had died within 12 months, or if 50 had died during the admission to hospital. Out of a total of 473 patients, nurses predicted that 180 would die within the next 12 months, and 74 would die during the admission. The actual survival figures were not reported [36]. Lilley et al. [39] reported on the accuracy of 28 clinicians who provided responses for 163 patients. Their results show a 'No' SQ

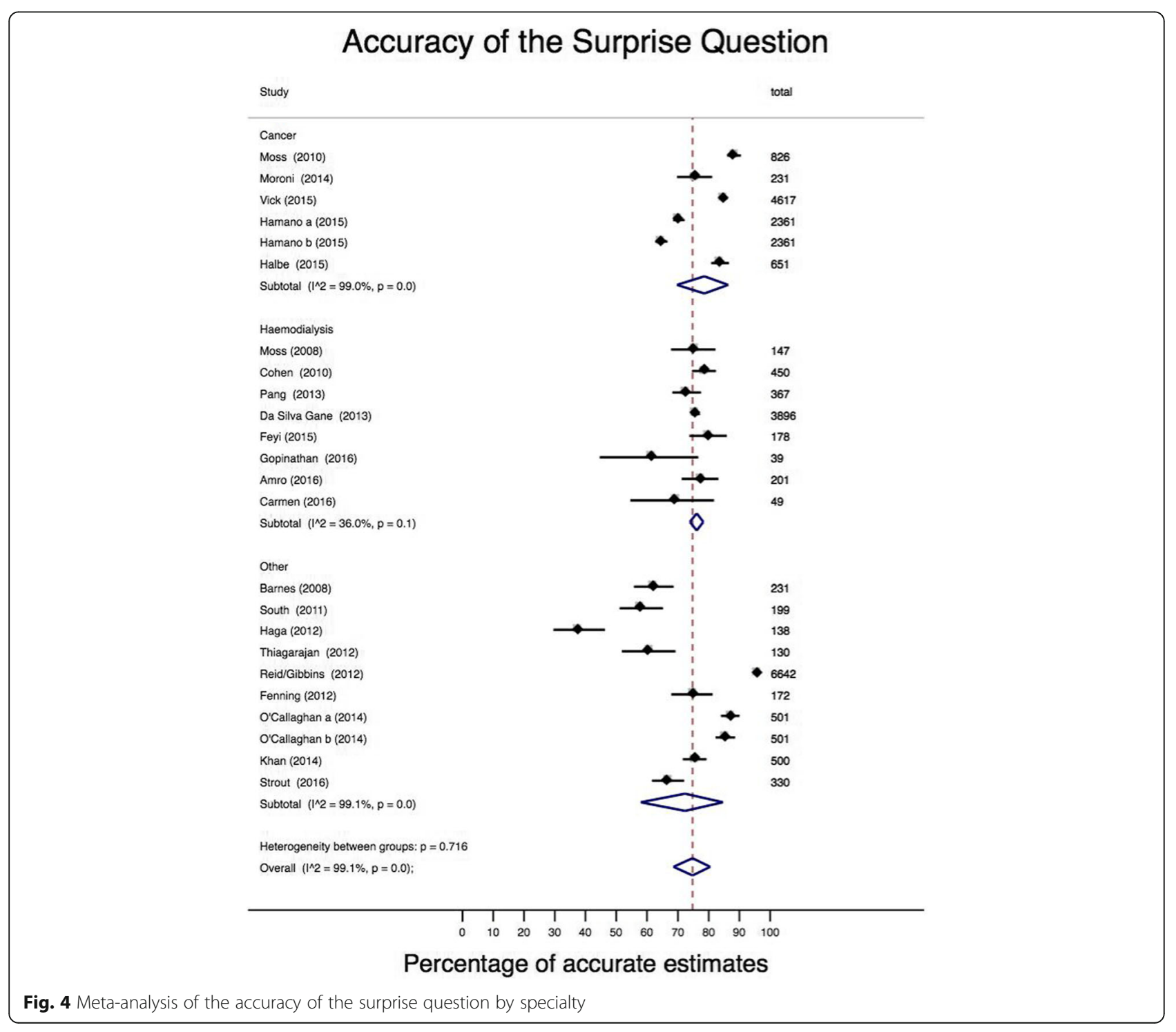


response was given in 93 cases $(60 \%)$. They reported a sensitivity of $81 \%$ (95\% CI $71-91 \%)$, a specificity of $51 \%$ (41-61\%), a PPV of $52 \%(42-61 \%)$, and a NPV of $82 \%$ (72-91\%). The exact breakdown of outcome by SQ prediction was not presented [39]. Lefkowits et al. [38] report the accuracy of the SQ within gynaecological oncology and across physicians. They asked 22 clinicians (18 gynaecologic oncology physicians and advanced practice providers (APP); four chemotherapy nurses) the SQ with a timeframe of 12 months. They reported the unadjusted odds ratio for death within a year associated with a 'No' SQ response; physicians $5.6(P<0.001)$, APP $9.2(P<0.001)$ and nurse $6.9(P<0.001)$. They reported that the APP group had the highest sensitivity (79.5\%) and the nurses had the best specificity (75.6\%). No further data was presented [38].

\section{Discussion}

The reported accuracy of the SQ varied considerably between studies. The c-statistic ranged from 0.512 (poor) to 0.822 (good). The PPV ranged from $13.9 \%$ to $78.6 \%$. This degree of heterogeneity was not uncommon in studies assessing the accuracy of clinicians' prognostic estimates [6].

On meta-analysis, the overall accuracy of the SQ was approximately $75 \%$. However, this overall estimate should be reviewed with caution given the low proportion of people who died within each study $(16 \%)$ and the low number of high quality studies included $\left(I^{2}=99 \%\right)$. There was virtually no difference in level of accuracy when considering studies in which the timeframe of the SQ had been reduced, which suggests that even when the patient is thought to be imminently dying, there is only moderate accuracy and continued uncertainty. A major limitation of the meta-regression analysis completed in this review was the lack of power due to the small sample size $(n=24)$. Therefore, a significant difference between time frames of the SQ was less likely to be observed.

One study presented data about the differences in accuracy between different professional groups at using the SQ. This suggested that doctors' responses to the SQ (c-statistic 0.735) may be more accurate than nurses' (cstatistic 0.632); however, more research is needed to fully address this question.

The variation in the accuracy of the SQ might be due as much to variations in disease trajectory in the last year of life as it is due to variations in the prognostic ability of the clinicians. There is some evidence that the SQ may be slightly better when used in oncology patients rather than in renal or other disease groups. The pooled accuracy for oncology was $79 \%$ compared to $76 \%$ for renal and $72 \%$ for other disease groups. This supports the idea that patients with a cancer diagnosis have a more predictable disease trajectory [45]. However, there was little variation between the disease groups and so these data should be interpreted cautiously. Another recent review on the SQ (using different inclusion and exclusion criteria to our own) [46] also found that accuracy was slightly better in oncology patients than in other disease groups.

When proposing the original definition of the SQ, Lynn suggested that the accuracy of the outcome was not actually that relevant as all patients identified by this question would typically need the services of palliative care such as advance care planning, home care assistance or financial support [13]. However, it is often the case that clinicians delay referring to palliative care services because they feel that the judgment should be made on the basis of a reasonably accurate and relatively short prognosis. This review highlights that, intuitively, clinicians are actually quite good at excluding patients with longer survival times but that use of the SQ alone is likely to lead to identification of a substantial number of patients who are not necessarily approaching the ends of their lives. However, the review could not provide evidence about how many patients who were identified (or missed) by the SQ actually had palliative care needs.

What is apparent from the data presented is that using the SQ to identify patients with a limited prognosis will detect at least as many 'false positives' as 'true positives'. In most circumstances, the consequences of misclassification by the SQ are rarely likely to be clinically important. For instance, erroneously including patients on a palliative care register who are not actually in the last year of life is unlikely to adversely affect their care, and indeed may result in better provision of holistic care. However, the recognition that half of patients included on such registers, as a result of the SQ, may not actually be in the last year of life has resource implications (e.g. additional staff time, care planning and multi-disciplinary involvement). It is thus not clear whether the SQ is a cost-effective way of identifying patients potentially suitable for palliative care. A careful balance is needed between identifying more people with unmet palliative care needs in a timely way while not over-burdening limited resources with too many patients in need of good care for long-term conditions over a much longer period.

It should be acknowledged that the SQ is usually used as part of a wider prognostic assessment that includes both general measures of performance status and disease burden along with disease-specific indicators $[1,2]$. It is possible that the combination of the SQ with these other prognostic measures may well be more accurate than the SQ used in isolation. This, however, was not the focus of this systematic review and further work is needed in order to evaluate the accuracy of these approaches and to determine whether other prognostic 
tools (e.g. Prognosis in Palliative Care Study [47], Palliative Prognostic Score [10] or Palliative Performance Scale [48]) would be a more accurate way of identifying patients approaching the end of life, or whether accuracy could be further improved by using the SQ alongside other tools such as the Palliative Outcome Scale, which can identify and document changes in the patient's condition over time [49].

Our study had a number of strengths. This was the first systematic review of the SQ that has attempted a meta-analysis of all studies reporting data on the SQ, including shorter time scales such as 7 days. We adopted a broad search strategy to identify any potentially relevant papers. We also obtained missing data by contacting relevant authors and requesting unpublished results. We have also appraised the quality of the papers included in our study and have conducted a sensitivity analysis to determine whether our conclusions are robust. Finally, each stage of the review process was undertaken by two independent reviewers to ensure rigor.

Our study had a number of limitations. It was difficult to devise a search strategy to capture all of the relevant papers. There is no agreed methodology for conducting a search of the literature for this type of research question. It is therefore possible that some papers may have been missed. In order to perform a meta-analysis of data, we combined studies that had evaluated the accuracy of the SQ over different time-frames, in widely differing patient groups and with different groups of clinicians. It may be argued that this is not a legitimate approach in terms of clinical heterogeneity as the performance of the SQ may be very different in each of these different circumstances. Further work is clearly needed to investigate any such differences (particularly our preliminary finding that a doctor's estimates may be more informative than nurses' estimates).

\section{Conclusion}

This review has highlighted the wide degree of accuracy reported for the SQ as a prognostic tool. Further work is required to understand the processes by which clinicians arrive at their prognostic estimates, to refine the accuracy of the SQ and to compare its performance against other more sophisticated prognostic tools, particularly in populations where a higher proportion of deaths occur.

\section{Additional files}

Additional file 1: Figure S1. Funnel plot to assess publication bias. (PNG $31 \mathrm{~kb}$ )

Additional file 2: Table S1. Studies that were excluded during the full review and the reason for exclusion. (DOCX $18 \mathrm{~kb}$ )

Additional file 3: Table S2. Quality rating using the Newcastle-Ottawa Scale. (DOCX 16 kb)

\section{Acknowledgements}

We would like to thank all of the corresponding authors of the papers listed within this systematic review. Their quick responses enable this review to be completed in a timely manner.

\section{Funding}

This study was funded as part of a UCL PhD Studentship and supported by Core Funding from Marie Curie.

\section{Availability of data and materials}

All data generated or analysed during this study are included in this published article and its supplementary information files.

\section{Authors' contributions}

NW developed the search terms, completed the data extraction, quality review, meta-analysis, and drafted the paper. NK developed the search terms, completed the data extraction, quality review, meta-analysis, and drafted the paper. W aided in the meta-analysis and drafted the paper. PS oversaw the development of the search terms and data extraction, aided in the metaanalysis, and drafted the paper. All authors approved the final version.

Ethics approval and consent to participate

Not applicable.

\section{Consent for publication}

Not applicable.

\section{Competing interests}

The authors declare that they have no competing interests.

\section{Publisher's Note}

Springer Nature remains neutral with regard to jurisdictional claims in published maps and institutional affiliations.

Received: 1 March 2017 Accepted: 3 July 2017

Published online: 02 August 2017

\section{References}

1. Thomas K, Armstrong Wilson J, GSF Team. Proactive Identification Guidance (PIG) National Gold Standards Framework Centre in End of Life Care. 2016. http://www.goldstandardsframework.org.uk/. Accessed 3 May 2017.

2. Weissman DE, Meier DE. Identifying patients in need of a palliative care assessment in the hospital setting a consensus report from the center to advance palliative care. J Palliat Med. 2011;14(1):17-23.

3. Murtagh FE, Bausewein C, Verne J, Groeneveld El, Kaloki YE, Higginson IJ. How many people need palliative care? A study developing and comparing methods for population-based estimates. Palliat Med. 2014;28(1):49-58.

4. Commissioning Guidance for Specialist Palliative Care: Helping to deliver commissioning objectives, December 2012. Guidance document published collaboratively with the Association for Palliative Medicineof Great Britain and Ireland, Consultant Nurse in Palliative Care Reference Group, Marie Curie Cancer Care, National Council for Palliative Care, and Palliative Care Section of the Royal Society of Medicine, London, UK.

5. Morrison RS, Augustin R, Souvanna P, Meier DE. America's care of serious illness: a state-by-state report card on access to palliative care in our nation's hospitals. J Palliat Med. 2011;14(10):1094-6.

6. White N, Reid F, Harris A, Harries P, Stone P. A systematic review of predictions of survival in palliative care: how accurate are clinicians and who are the experts? PLOS ONE. 2016;11(8), e0161407.

7. Glare P, Virik K, Jones M, Hudson M, Eychmuller S, Simes J, et al. A systematic review of physicians' survival predictions in terminally ill cancer patients. BMJ. 2003;327(7408):195.

8. Hui D, Kilgore K, Nguyen L, Hall S, Fajardo J, Cox-Miller TP, et al. The accuracy of probabilistic versus temporal clinician prediction of survival for patients with advanced cancer: a preliminary report. Oncologist. 2011;16: 1642-8.

9. Neuberger J, Guthrie C, Aaronovitch D. More care, less pathway: a review of the Liverpool Care Pathway. Department of Health. 2013.

10. Pirovano M, Maltoni M, Nanni O, Marinari M, Indelli M, Zaninetta G, et al. A new palliative prognostic score: a first step for the staging of terminally ill cancer patients. J Pain Symptom Manag. 1999;17(4):231-9. 
11. Morita T, Tsunoda J, Inoue S, Chihara S. The palliative prognostic index: a scoring system for survival prediction of terminally ill cancer patients. Support Care Cancer. 1999;7:128-33.

12. British Medical Association. End-of-Life Care and Physician-Assisted Dying. 2016. https://www.bma.org.uk/collective-voice/policy-and-research/ethics/ end-of-life-care. Accessed 3 May 2017

13. Lynn J. Living long in fragile health: the new demographics shape end of life care. Hast Cent Rep. 2005;35(7):s14-8.

14. National Institute for Health and Care Excellence (NICE). End of Life Care for Adults. 2011. Updated 2013. https://www.nice.org.uk/guidance/qs13. Accessed 3 May 2017

15. Haga K, Murray S, Reid J, Ness A, O'Donnell M, Yellowlees D, et al. Identifying community based chronic heart failure patients in the last year of life: A comparison of the Gold Standards Framework Prognostic Indicator Guide and the Seattle Heart Failure Model. Heart. 2012;98(7):579-83.

16. Wells $G$, Shea B, O'connell D, Peterson J, Welch V, Losos M, et al. The Newcastle-Ottawa Scale (NOS) for assessing the quality of nonrandomised studies in meta-analyses. 2000. Ottawa, Ontario The Ottawa Health Research Institute: [01/09/2016]; Available from: http://www.ohri.ca/programs/clinical_ epidemiology/oxford.asp

17. Diagnostic Test Studies: Assessment and Critical Appraisal. BMJ Clinical Evidence. 2014.

18. Amro OW, Ramasamy M, Strom JA, Weiner DE, Jaber BL. Nephrologistfacilitated advance care planning for hemodialysis patients: a quality improvement project. Am J Kidney Dis. 2016;68(1):103-9.

19. Carmen JM, Santiago P, Elena D, Silvia S, Pedro MJ, Jesus LP, et al. Frailty, surprise question and mortality in a hemodialysis cohort question and mortality in a hemodialysis cohort. Nephrol Dial Transplant. 2016;31 Suppl 1:1553.

20. Cohen LM, Ruthazer R, Moss AH, Germain MJ. Predicting six-month mortality for patients who are on maintenance hemodialysis. Clin J Am Soc Nephrol. 2010;5(1):72-9.

21. Feyi K, Klinger S, Pharro G, McNally L, James A, Gretton K, et al. Predicting palliative care needs and mortality in end stage renal disease: use of an atrisk register. BMJ Support Palliat Care. 2015:5(1):19-25.

22. Halbe L, Gerlach C, Hess G, Wehler T, Theobald M, Weber M. "Would I be surprised if this patient died in the next year?" - Prognostic significance of the "Surprise" Question in a university hematology and oncology outpatients clinic. Jahrestagung der Deutschen, Osterreichischen und Schweizerischen Gesellschaften fur Hamatologie und Medizinische Onkologie. Basel: S. Karger AG; 2015

23. Hamano J, Morita T, Inoue S, Ikenaga M, Matsumoto Y, Sekine E, et al. Surprise questions for survival prediction in patients with advanced cancer: a multicenter prospective cohort study. Oncologist. 2015;20(7):839-44.

24. Khan M, Abdulnabi K, Pai P. A staff survey on end of life care in advanced kidney disease. 49th ERA-EDTA Congress Paris France. Oxford: Oxford University Press; 2012. p. ii281-2.

25. Moroni M, Zocchi D, Bolognesi D, Abernethy A, Rondelli R, Savorani G, et al The 'surprise' question in advanced cancer patients: a prospective study among general practitioners. Palliat Med. 2014;28(7):959-64.

26. Moss A, Lunney J, Culp S, Abraham J. Prognostic significance of the surprise question in cancer patients. Annual Assembly of the American Academy of Hospice and Palliative Medicine (AAHPM) and the Hospice and Palliative Nurses Association (HPNA). Boston, MA: AAHPM; 2010. p. 346

27. Moss AH, Ganjoo J, Sharma S, Gansor J, Senft S, Weaner B, et al. Utility of the "Surprise" question to identify dialysis patients with high mortality. Clin J Am Soc Nephrol. 2008;3(5):1379-84.

28. O'Callaghan A, Laking G, Frey R, Robinson J, Gott M. Can we predict which hospitalised patients are in their last year of life? A prospective crosssectional study of the Gold Standards Framework Prognostic Indicator Guidance as a screening tool in the acute hospital setting. Palliat Med. 2014; 28(8):1046-52.

29. Pang WF, Kwan BC, Chow KM, Leung CB, Li PK, Szeto CC. Predicting 12 month mortality for peritoneal dialysis patients using the "surprise" question. Perit Dial Int. 2013;33(1):60-6.

30. Fenning S, Woolcock R, Haga K, lqbal J, Fox KA, Murray SA, et al. Identifying acute coronary syndrome patients approaching end-of-life. PLoS ONE. 2012; 7(4), e35536.

31. Gopinathan J, Aboobacker I, Hafeeq B, Aziz F, Narayanan R, Narayanan S. Predicting death on maintenance hemodialysis - a complex task in prevalent elders. 53rd ERA-EDTA Congress, Vienna, Austria. Oxford: Oxford University Press; 2016. p. i550.
32. South $G$, Reddington $O$, Hatfield L, Phillips A, Wall H. End of life in COPD: There may be no surprises! European Respiratory Society Annual Congress. Amsterdam: European Respiratory Society; 2011.

33. Thiagarajan R, Morris J, Harkins KJ. Can simple intuitive questions identify patients in the last year of their life?-a pragmatic study comparing the "paired surprise questions" with the "single surprise question". Brighton: British Geriatrics Society Autumn Meeting; 2012. p. i61.

34. Vick J, Pertsch N, Hutchings M, Neville B, Bernacki R. The utility of the surprise question in identifying patients most at risk of death, Annual Assembly of the American Academy of Hospice and Palliative Medicine and the Hospice and Palliative Nurses Association. Chicago, IL: Elsevier Inc.; 2016. p. 342.

35. Barnes S, Gott M, Payne S, Parker C, Seamark D, Gariballa S, et al. Predicting mortality among a general practice-based sample of older people with heart failure. Chron IIIn. 2008:4(1):5-12.

36. Gardiner C, Gott M, Ingleton C, Seymour J, Cobb M, Noble B, et al. Extent of palliative care need in the acute hospital setting: A survey of two acute hospitals in the UK. Palliat Med. 2013;27(1):76-83.

37. Johnson M, Nunn A, Hawkes T, Stockdale S, Daley A. Planning for end-of-life care in heart failure: Experience of two integrated cardiology-palliative care teams. Br J Cardiol. 2012;19(2):71-5.

38. Lefkowits C, Chandler C, Sukumvanich P, Courtney-Brooks M, Duska L, Althouse A, et al. Validation of the 'surprise question' in gynecologic oncology: comparing physicians, advanced practice providers, and nurses. 47th Annual Meeting on Women's Cancer of the Society of Gynecologic Oncology. San Diego, CA: Society of Gynecologic Oncology; 2016.

39. Lilley EJ, Gemunden SA, Kristo G, Changoor N, Scott JW, Rickerson E, et al. Utility of the "surprise" question in predicting survival among older patients with acute surgical conditions. J Palliat Med. 2016;1:1.

40. Da Silva GM, Braun A, Stott D, Wellsted D, Farrington K. How robust is the 'surprise question' in predicting short-term mortality risk in haemodialysis patients? Nephron Clin Pract. 2013;123(3-4):185-93.

41. Gibbins J, Reid C, Bloor S, Burcombe M, McCoubrie R, Forbes K. The use of a modified 'surprise' question to identify and recruit dying patients into a research project. 7th World Research Congress of the European Association for Palliative Care. Trondheim: EAPC; 2012. p. 418-9.

42. Strout TDS, Haydar SA, Eager E, Han PKJ. Identifying unmet palliative care needs in the ED: Use of the 'surprise question' in patients with sepsis. Annual Meeting of the Society for Academic Emergency Medicine. New Orleans, LA: SAEM; 2016. p. S196.

43. Reid CM, Gibbins J, Bloor S, Burcombe M, McCoubrie R, Forbes K. Can the impact of an acute hospital end-of-life care tool on care and symptom burden be measured contemporaneously? BMJ Support Palliat Care. 2013; 3(2):161-7.

44. Khan S, Hadique S, Culp S, Syed A, Hodder C, Parker J, et al. Efficacy of the "surprise" question to predict 6-month mortality in ICU patients. Phoenix, AZ: Critical Care Congress; 2014. p. A1457.

45. Murray SA, Kendall M, Boyd K, Sheikh A. Illness trajectories and palliative care. Int Perspect Public Health Palliat Care. 2012;30:2017-9.

46. Downar J, Goldman R, Pinto R, Englesakis M, Adhikari NK. The "surprise question" for predicting death in seriously ill patients: a systematic review and meta-analysis. Can Med Assoc J. 2017;189(13):E484-93.

47. Gwilliam B, Keeley V, Todd C, Gittins M, Roberts C, Kelly L, et al. Development of prognosis in palliative care study (PiPS) predictor models to improve prognostication in advanced cancer: prospective cohort study. BMJ. 2011;343:d4920.

48. Anderson F, Downing GM, Hill J, Casorso L, Lerch N. Palliative performance scale (PPS): a new tool. J Palliat Care. 1996;12(1):5-11.

49. Palliative Care Outcome Scale. 2012. https://pos-pal.org. Accessed 3 May 2017. 